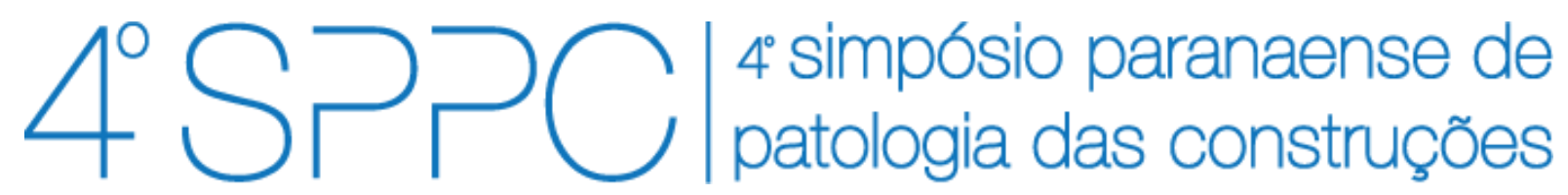

ISSN 2526-7248 artigo n. 4SPPC138, pp. 338-347, 2019

\title{
Abrasão superficial de concretos contendo cinza volante em substituição parcial ao cimento Portland
}

\author{
Witzke, Franky Bruno ${ }^{1}$; Correia, Sivaldo Leite ${ }^{2}$; Medeiros-Junior, Ronaldo Alves ${ }^{3}$ \\ ${ }^{1}$ Doutorando em Engenharia de Construção Civil, UFPR, fwitzke@hotmail.com \\ 2 Professor Doutor, Departamento de Química, UDESC, sivaldo.leite@gmail.com \\ ${ }^{3}$ Professor Doutor, Departamento de Construção Civil, UFPR, \\ medeirosjunior.ufpr@gmail.com
}

Resumo: $O$ presente artigo tem por objetivo investigar a abrasão superficial e a resistência à compressão de concretos contendo cinza volante. Foi utilizado um planejamento fatorial fracionado para o desenvolvimento do trabalho, englobando nove misturas com diferentes relações água/aglomerante (A/A: 0,28; 0,33 e 0,40), agregado/aglomerante (AG/A: 2,70; 3,10 e 3,70) e teores de substituição de cimento Portland por cinza volante (TC:10, 20 e 30\%). Foram testadas a resistência à compressão aos 28 dias de cura (RC28), bem como a resistência à abrasão superficial dos concretos (RAS). Os resultados mostraram que é possível se obter concretos com cinza volante com alta resistência à compressão e perda mínima do desgaste abrasivo. Dessa forma, o uso de cinza volante em concretos pode ser uma alternativa para a substituição parcial do cimento Portland, do ponto de vista das propriedades estudadas.

Palavras-chave: Concreto, abrasão superficial, cinza volante.

Abstract: The present article aims to investigate the superficial abrasion resistance and the compressive strength of fly ash concretes. A fractionated factorial design was used for the development of the work, using nine different mixtures with water/binder contents (A/A: 0,28, 0,33 and 0,40), aggregate/binder (AG/A: 2,70, 3,10 and 3,70 ) and the substitution content of Portland cement by fly ash (TC: 10, 20 and $30 \%$ ). The compressive strength was tested at 28 days of curing (RC28) as well as the surface abrasion resistance of the concretes (RAS). The results showed that is possible to obtain fly ash concretes with high compressive strength and minimum loss of material in surface by abrasion. Thus, the use of fly ash in concrete may be an alternative for the partial replacement of Portland cement from the point of view of the properties studied.

Keywords: Concrete, superficial abrasion, fly ash. 
WITZKE, FRANKY BRUNO; CORREIA, SIVALDO LEITE, MEDEIROS-JUNIOR; RONALDO ALVES. ABRASÃO SUPERFICIAL DE CONCRETOS CONTENDO CINZA VOLANTE EM SUBSTITUIÇÃO PARCIAL AO CIMENTO PORTLAND. $4^{\circ}$ Simpósio Paranaense de Patologia das Construções (40 SPPC), artigo 4SPPC138, pp. 338 - 347, 2019. DOI: 10.4322/2526-7248.056

\section{Introdução}

O concreto é um material mundialmente utilizado e é resultado do desenvolvimento dos seus componentes, como cimento Portland, proporcionando crescimento do setor da construção civil, e da economia global [1-5]. A indústria cimenteira, porém, é considerada como grande poluidora do meio ambiente, além de ser responsável por cerca de $7 \%$ do total de emissões de $\mathrm{CO}_{2}$ na atmosfera. O setor consome, ainda, grandes quantidades de energia durante o processo de fabricação de seus componentes [6-8].

Apesar disso, problemas relacionados à resistência e durabilidade do material não são raros e impõem elevados custos em sua reparação no mundo inteiro [9]. Em muitos locais, os aportes financeiros gastos com a recuperação de estruturas de concreto são maiores do que aqueles utilizados na construção de novas edificações [10].

Dessa forma, existe uma busca, por parte da indústria e da ciência, por materiais, técnicas e processos que visem minimizar os impactos ambientais e melhorar o desempenho dos materiais de construção, bem como o do concreto, do ponto de vista da durabilidade.

Muitas pesquisas têm mostrado que a utilização de adições minerais e resíduos industriais com características pozolânicas, tais como a cinza volante, são benéficas nas propriedades do concreto, melhorando as características mecânicas e também aumentando a durabilidade, na maioria das situações. Além disso, ao substituir o cimento Portland pelas adições minerais ou resíduos industriais, é possível reduzir o uso de energia e a emissão de gases na atmosfera. Outro aspecto positivo é o emprego de um material que poderia ser disposto em aterros sanitários, sem perspectivas de reutilização. O custo do concreto com adições minerais também é reduzido, tendo em vista que o cimento Portland é o componente mais caro do concreto $[1 ; 11-19]$.

A abrasão superficial do concreto é a perda progressiva de massa da superfície que ocorre por meio seco, sendo comum em pavimentos industriais e em locais com tráfego de veículos. Esse desgaste pode ocorrer por fricção, raspagem, derrapagem ou deslizamento de objetos na superfície do material $[1 ; 20]$. É, ainda, uma das formas mais comuns de degradação do concreto e está ligada a diversos fatores internos e controláveis da massa de concreto, tais como a relação água/aglomerante $(\mathrm{A} / \mathrm{A})$, tipo e qualidade dos agregados, bem como a utilização de adições minerais. Além disso, técnicas construtivas e incorreto manuseio, entre outros fatores incontroláveis, também acarretam em mudanças na propriedade [2122].

Dentro deste contexto, o objetivo deste trabalho foi avaliar a resistência à abrasão superficial de misturas de concreto que contém cinza volante como substituição parcial ao cimento Portland. Para tanto, foi desenvolvido um projeto fatorial fracionado com nove misturas diferentes de concreto, variando em três níveis a relação água/aglomerante, agregado/aglomerante e teor de substituição de cinza volante pelo cimento Portland. Em complemento, a resistência à compressão também foi investigada. 


\section{Materiais e métodos}

\subsection{Materiais}

Os agregados graúdos e miúdos utilizados foram a brita tamanho 0 e a areia média, ambos extraídos da região nordeste do estado de Santa Catarina. O cimento Portland utilizado foi do tipo CP II-Z 32, por ser amplamente comercializado em Santa Catarina e pela sua versatilidade de utilização. A cinza volante é originária da Usina Termelétrica Jorge Lacerda, da cidade de Capivari de Baixo, sul do estado de Santa Catarina, fornecida pela empresa Engie Energia. A Tabela 1 mostra a composição química da cinza volante.

Tabela 1: Composição química da cinza volante

\begin{tabular}{ccccccccc}
\hline Elemento & $\mathrm{SiO}_{2}$ & $\mathbf{A l}_{2} \mathrm{O}_{3}$ & $\mathrm{Fe}_{2} \mathrm{O}_{3}$ & $\mathbf{K}_{2} \mathrm{O}$ & $\mathbf{C a O}$ & $\mathrm{TiO}_{2}$ & $\mathbf{S O}_{3}$ & $\mathbf{P}_{2} \mathrm{O}_{5}$ \\
\hline Conteúdo (\%) & 51,25 & 31,87 & 7,08 & 3,99 & 2,05 & 1,56 & 1,49 & 0,70
\end{tabular}

\subsection{Procedimento experimental}

Foi realizado um estudo preliminar para obtenção de valores de relação água/aglomerante $(A / A)$, agregado/aglomerante $(A G / A)$ e teor de substituição decinza volante por cimento Portland (TC). Com base no estudo de Buest, Silva e Campitelli [23], foram adotados parâmetros para um concreto com resistência à compressão de dosagem $\left(f_{c k}\right)$ após 28 dias de cura de $30 \mathrm{MPa}$ e para uma trabalhabilidade de $100 \mathrm{~mm}$. Foi utilizado, então, um projeto fatorial fracionado $3^{3-1}$, para o planejamento experimental, que permitiu o planejamento e a realização de maneira organizada da quantidade mínima necessária de experimentos, com economia de tempo e de recursos financeiros. Utilizou-se o software Statistica 8.0 [24] para a obtenção de 9 (nove) misturas diferentes, delineadas aleatoriamente. As misturas são apresentadas na Tabela 2.

Tabela 2: Misturas de concreto do projeto fatorial fracionado

\begin{tabular}{cccc|cccc}
\hline Misturas & A/A & AG/A & TC (\%) & Misturas & A/A & AG/A & TC (\%) \\
\hline Padrão & 0,33 & 3,10 & 0 & 5 & 0,33 & 3,10 & 20 \\
1 & 0,28 & 2,70 & 10 & 6 & 0,33 & 3,60 & 10 \\
2 & 0,28 & 3,10 & 30 & 7 & 0,40 & 2,70 & 20 \\
3 & 0,28 & 3,60 & 20 & 8 & 0,40 & 3,10 & 10 \\
4 & 0,33 & 2,70 & 30 & 9 & 0,40 & 3,60 & 30 \\
\hline
\end{tabular}

A moldagem dos corpos de prova ocorreu de acordo com a NBR 5738 [25]. Os corpos de prova foram desmoldados e mantidos sob cura úmida, a $25^{\circ} \mathrm{C}$, até a realização dos ensaios. Foi realizado um ensaio de resistência à compressão aos 28 dias de cura e também um de resistência à abrasão superficial [26]. No final foi realizada uma correlação entre os dois ensaios, em um processo de otimização das misturas de concreto. 
O ensaio de resistência à compressão aos 28 dias de cura foi realizado com 0 auxílio de uma prensa hidráulica elétrica, conectada a um computador, modelo Emic PC200l, instalada no Laboratório de Materiais de Construção do CCT/UDESC, foram utilizados três corpos de prova $(100 \mathrm{~mm} \times 200 \mathrm{~mm})$ para cada mistura de concreto

O ensaio de resistência à abrasão superficial foi desenvolvido pelo Centro de Ciência e Tecnologia do Rio Grande do Sul - CIENTEC [26] e trata-se de uma metodologia para a simulação do desgaste por abrasão, constituindo na simulação de um percurso de $500 \mathrm{~m}$ percorridos por dois corpos de prova de $50 \times 50 \mathrm{~mm}$, de cada mistura, a uma pressão de $0,06 \mathrm{MPa}$. O material abrasivo utilizado foi 0 carbeto de silício e o resultado foi mensurado pela média do desgaste em cinco pontos do corpo de prova, em $\mathrm{mm}$.

A caracterização das misturas de concreto foi realizada por meio da análise estatística do software Statistica 8.0 [24]. A partir da significância e adequação, modelos de regressão foram obtidos para cada propriedade, como uma função dos fatores A/A, AG/A e TC. Com base nos modelos obtidos, foi utilizada a técnica de metodologia de superfícies de resposta (MSR) para avaliar o efeito nas propriedades.

\section{Resultados e discussões}

A Tabela 3 apresenta os resultados para a resistência à compressão (média e desvio padrão) e resistência à abrasão superficial (profundidade em mm).

Tabela 3: Resultados de RC28 e RAS

\begin{tabular}{|c|c|c|c|c|c|}
\hline Misturas & A/A & AG/A & TC (\%) & RC28 & RAS \\
\hline Padrão & 0,33 & 3,10 & 0 & (MPa) & $(\mathrm{mm})$ \\
\hline 1 & 0,28 & 2,70 & 10 & $32,46 \pm 5,64$ & 5,26 \\
\hline 2 & 0,28 & 3,10 & 30 & $25,77 \pm 0,72$ & 5,24 \\
\hline 3 & 0,28 & 3,60 & 20 & $39,68 \pm 2,25$ & 5,24 \\
\hline 4 & 0,33 & 2,70 & 30 & $37,29 \pm 2,30$ & 6,14 \\
\hline 5 & 0,33 & 3,10 & 20 & $37,15 \pm 3,49$ & 5,36 \\
\hline 6 & 0,33 & 3,60 & 10 & $34,09 \pm 2,11$ & 5,56 \\
\hline 7 & 0,40 & 2,70 & 20 & $31,34 \pm 2,25$ & 4,46 \\
\hline 8 & 0,40 & 3,10 & 10 & $31,28 \pm 3,51$ & 6,36 \\
\hline 9 & 0,40 & 3,60 & 30 & $30,05 \pm 1,35$ & 6,34 \\
\hline
\end{tabular}

\subsection{Resistência à compressão (RC28)}

Para compreensão e avaliação das interações entre as variáveis (quando existir), o uso da técnica de metodologia de superfícies de resposta (MSR) pode ser utilizada para estimar o efeito dessas variáveis na resposta. A MSR utiliza ferramentas estatísticas de significância e análise de regressão para a obtenção do melhor 
WITZKE, FRANKY BRUNO; CORREIA, SIVALDO LEITE, MEDEIROS-JUNIOR; RONALDO ALVES. ABRASÃO SUPERFICIAL DE CONCRETOS CONTENDO CINZA VOLANTE EM SUBSTITUIÇÃO PARCIAL AO CIMENTO PORTLAND. $4^{\circ}$ Simpósio Paranaense de Patologia das Construções (40 SPPC), artigo 4SPPC138, pp. 338 - 347, 2019. DOI: 10.4322/2526-7248.056

modelo matemático que represente o efeito dos fatores (as variáveis) nas propriedades. Muitos trabalhos desenvolvidos em pesquisas na UDESC e em outros locais tratam dos procedimentos relacionados com essa técnica [2; 17; 27-28]

A Figura 1 apresenta a superfície de resposta para o efeito das variáveis TC e A/A para o valor de RC28, mantendo a AG/A constante em 3,10, valor médio entre as relações AG/A e o de menor significância para obtenção de RC28.

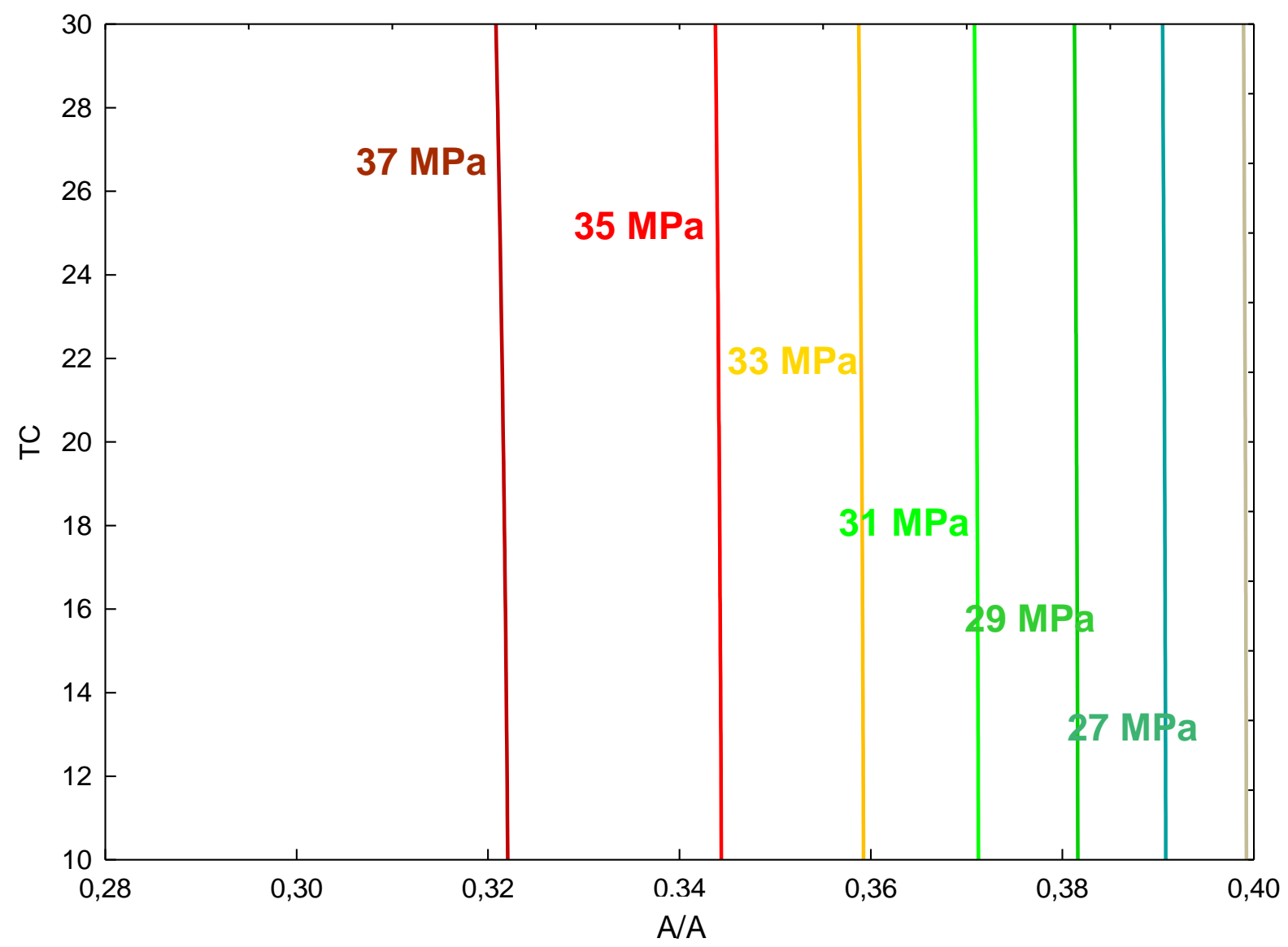

Figura 1: Gráfico de contorno de linhas para RC28 das misturas de concreto, como uma função de A/A e TC, mantendo-se constante AG/A em 3,10.

A Figura 1 mostra que o TC não afeta a RC28, a qual é fortemente dependente da A/A. A partir da Figura 1 é possível obter parâmetros para a produção de concretos com resistência superior a $29 \mathrm{MPa}$ após 28 dias de cura, usando relações A/A inferiores a 0,38 , em quaisquer valores de substituição de cimento Portland por cinza volante, dentro dos padrões estudados (mantendo a AG/A constante em 3,10 ). Com uma menor A/A, a matriz gera menor porosidade, contribuindo, assim, para uma maior RC28 [1]. 
WITZKE, FRANKY BRUNO; CORREIA, SIVALDO LEITE, MEDEIROS-JUNIOR; RONALDO ALVES. ABRASÃO SUPERFICIAL DE CONCRETOS CONTENDO CINZA VOLANTE EM SUBSTITUIÇÃO PARCIAL AO CIMENTO PORTLAND. $4^{\circ}$ Simpósio Paranaense de Patologia das Construções (40 SPPC), artigo 4SPPC138, pp. 338 - 347, 2019. DOI: 10.4322/2526-7248.056

\subsection{Resistência à abrasão superficial (RAS)}

A Figura 2 apresenta a superfície de resposta para o efeito das variáveis TC e A/A para o valor de RAS, mantendo a AG/A constante em 3,10.

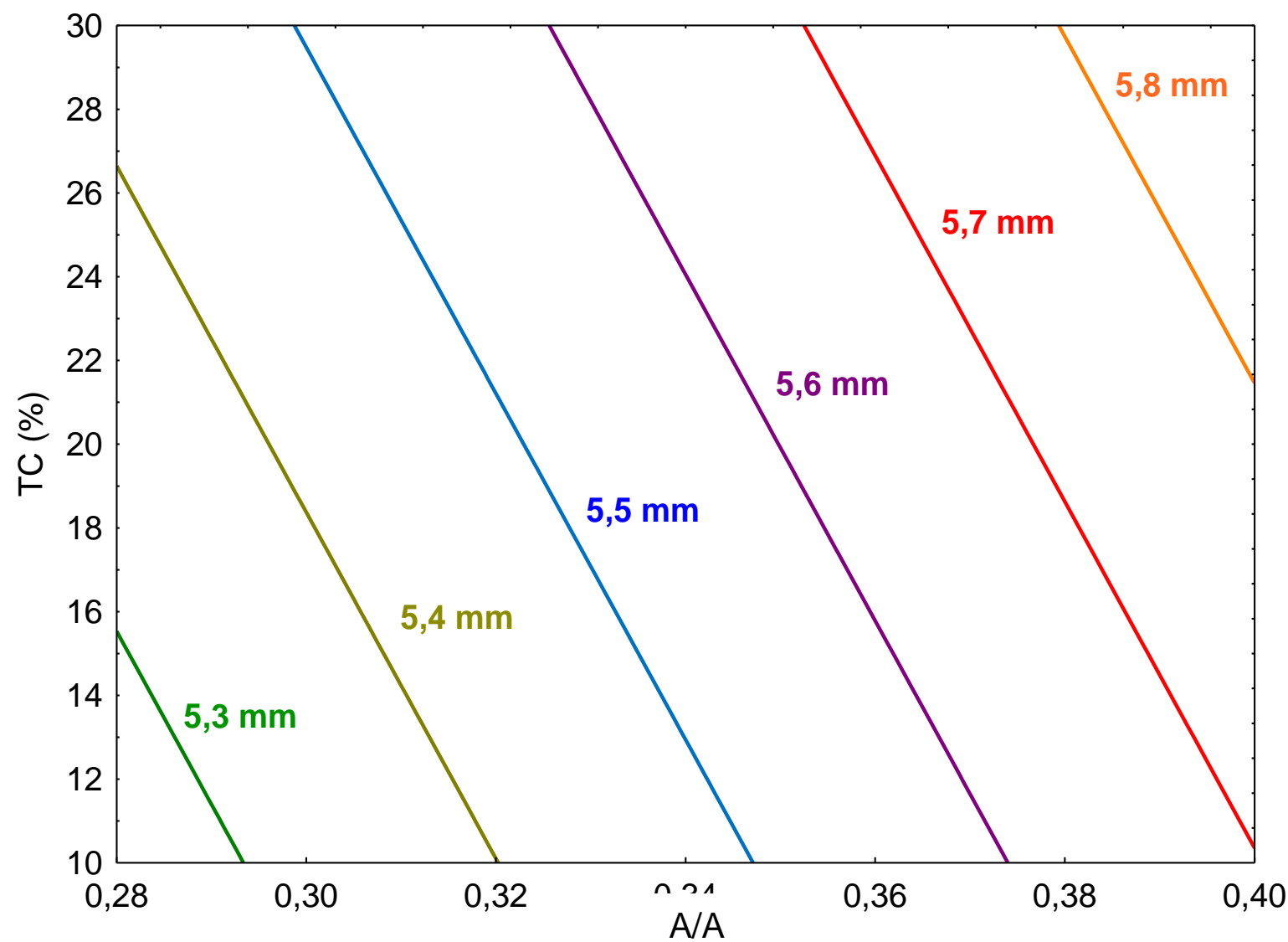

Figura 2: Gráfico de contorno de linhas para RAS das misturas de concreto, como uma função de A/A e TC, mantendo-se constante AG/A em 3,10.

A Figura 2 mostra que tanto TC quanto A/A influenciam a RAS. E é possível obter parâmetros para a produção de concretos com desgaste superficial inferior a 5,6 $\mathrm{mm}$, usando quaisquer valores para TC e A/A inferior a 0,37, dentro dos padrões estudados (mantendo a AG/A constante em 3,10 ). $O$ resultado vai ao encontro a outras pesquisas que mencionam que concretos com maior relação A/A e TC apresentam maior desgaste por abrasão [29]. 
WITZKE, FRANKY BRUNO; CORREIA, SIVALDO LEITE, MEDEIROS-JUNIOR; RONALDO ALVES. ABRASÃO SUPERFICIAL DE CONCRETOS CONTENDO CINZA VOLANTE EM SUBSTITUICCÃO PARCIAL AO CIMENTO PORTLAND. $4^{\circ}$ Simpósio Paranaense de Patologia das Construções (40 SPPC), artigo 4SPPC138, pp. 338 - 347, 2019. DOI: 10.4322/2526-7248.056

\subsection{Correlação entre ensaios - otimização de misturas}

A Figura 3 apresenta a superfície de resposta para o efeito das variáveis TC e A/A para o valor de RC28 e RAS, mantendo a AG/A constante em 3,10.

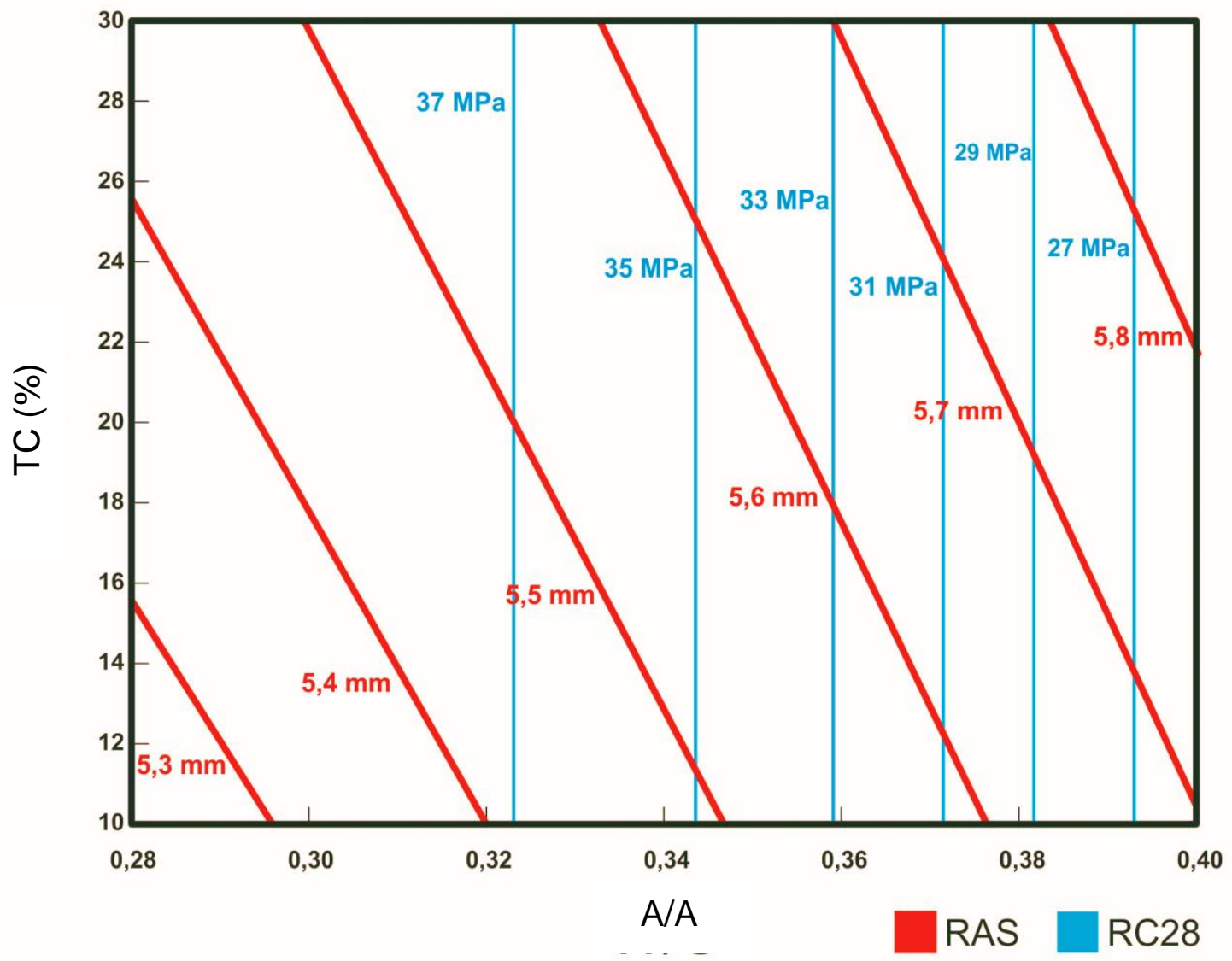

Figura 3: Gráfico de contorno de linhas para RC28 e RAS das misturas de concreto, como uma função de A/A e TC, mantendo-se constante AG/A em 3,10.

A Figura 3 mostra que é possível obter parâmetros para a produção de concretos com desgaste superficial inferior a 5,6 mm, com resistência à compressão superior a $31 \mathrm{MPa}$ usando quaisquer valores para TC e A/A inferiores a 0,37, dentro dos padrões estudados (mantendo a $A G / A$ constante em 3,10 ). $O$ resultado mostra também que há uma interligação entre as duas propriedades, e quanto maior a resistência à compressão, menor o desgaste abrasivo superficial. Comportamento também encontrado em outros estudos [29]. 
WITZKE, FRANKY BRUNO; CORREIA, SIVALDO LEITE, MEDEIROS-JUNIOR; RONALDO ALVES. ABRASÃO SUPERFICIAL DE CONCRETOS CONTENDO CINZA VOLANTE EM SUBSTITUIÇÃO PARCIAL AO CIMENTO PORTLAND. $4^{\circ}$ Simpósio Paranaense de Patologia das Construções (40 SPPC), artigo 4SPPC138, pp. 338 - 347, 2019. DOI: 10.4322/2526-7248.056

\section{Considerações finais}

A utilização de um projeto fatorial fracionado permitiu avaliar, ao mesmo tempo, os efeitos dos parâmetros de água/aglomerante, agregado/aglomerante e teor de cinza volante em substituição ao cimento Portland.

A resistência à compressão aos 28 dias de cura foi influenciada pela relação água/aglomerante, independente do teor de cinza volante.

Por outro lado, a resistência à abrasão superficial foi influenciada tanto pela relação água/aglomerante, quanto pelo teor de substituição de cinza volante pelo cimento Portland.

O processo de otimização mostrou que é possivel a obtenção de misturas de concreto com $37 \mathrm{MPa}$ de resistência a compressão e desgaste superficial por abrasão inferior a 5,4 mm, adotando-se A/A inferior a 0,32, TC entre 10 e $26 \%$ e AG/A de 3,1.

\section{Referências}

[1] MEHTA, P. Kumar; MONTEIRO, Paulo J.M.. Concreto: Microestrutura, propriedades e materiais. 2. ed. São Paulo: IBRACON, 2014.

[2] SCHWAAB, Samoel. Efeito da Adição de Aditivo Plastificante Retardador de Pega nas Propriedades de Concretos Usinados de Cimento Portland Usando Planejamento Fatorial. Dissertação (Mestrado em Ciência e Engenharia de Materiais) Centro de Ciências Tecnológicas - CCT. 2015. Joinville.

[3] PEDROSO, Fábio Luís. Concreto: as Origens e a Evolução do Material Construtivo mais. Usado pelo Homem. Concreto e Construções. v. 37, n. 53, p. 14-19, 2009.

[4] LIMA, J.A.R. Avaliação das consequências da produção de concreto no Brasil para as mudanças climáticas. 2010. 151 f. Tese (Doutorado em Engenharia). Escola Politécnica da Universidade de São Paulo. São Paulo, 2010.

[5] SHEN, Weiguo. et al. Mixing Design and Microstructure of Ultra High Strength Concrete with Manufactured Sand. Construction and Building Materials. v. 143, n. 14, p. 312-321, 2017.

[6] AlIABDO, Ali A.; ElMOATY, Adb Elmoaty M. Abd Elmoaty; SALEM, Hazem A. Effect of Cement Addition, Solution Resting Time and Curing Characteristics on Fly Ash Based Geopolymer Concrete Performance. Construction and Building Materials. v. 123, n. 19, p.581-593, 2016.

[7] RAKHIMOV, Ravil Z. et al. Properties of Portland Cement Pastes Enriched with Addition of Calcined Marl. Journal of Building Engineering. v. 11, n. 3, p. 30-36, 2017.

[8] CAMOES, Aires. Betões de Elevado Desempenho com Elevado Volume de Cinzas Volantes. Engenharia Civil. v. 10, n. 23, p. 55-64, 2005. 
WITZKE, FRANKY BRUNO; CORREIA, SIVALDO LEITE, MEDEIROS-JUNIOR; RONALDO ALVES. ABRASÃO SUPERFICIAL DE CONCRETOS CONTENDO CINZA VOLANTE EM SUBSTITUIÇÃO PARCIAL AO CIMENTO PORTLAND. $4^{\circ}$ Simpósio Paranaense de Patologia das Construções (40 SPPC), artigo 4SPPC138, pp. 338 - 347, 2019. DOI: 10.4322/2526-7248.056

[9] MEDEIROS JUNIOR, Ronaldo Alves de; LIMA, Maryangela Geimba de; BALESTRA, Carlos Eduardo Tino. Identificação das Principais Manifestações Patológicas em Estruturas de Concreto Armado Pertencentes ao DCTA. In: Congresso Internacional sobre Patologia e Reparação de Estruturas. 9. 2013. João Pessoa. Anais... João Pessoa: IFPB. p. 1 - 9.

[10] MA, Chau-Khun. et al. Repair and Rehabilitation of Concrete Structures Using Confinement: A Review. Construction and Building Materials. v. 133, n. 4, p. 502-515, 2017.

[11] CEZAR, Daiana de Souza. Características de Durabilidade de Concretos com Cinas Volante e Cinza de Casca de Arroz com e sem Beneficiamento. 2011. 143 f. Dissertação (Mestrado em Engenharia Civil) Centro de Tecnologia. Santa Maria. 2011.

[12] CANTARELLI, Gustavo Martins. Percolação de Água em Protótipos de Concreto com Elevados Teores de Escória e Cinza Volante com Adição de Cal. 2007. 107 f. Dissertação (Mestrado em Engenharia Civil) Centro de Tecnologia. Santa Maria. 2007.

[13] SACILOTO, Adriano Pedroso. Comportamento Frente à Ação de Cloretos de Concretos Compostos com Adições Minerais Submetidos a Diferentes Períodos de Cura. 200590 f. Dissertação (Mestrado em Engenharia Civil). Centro de Ciências Rurais. Santa Maria. 2005.

[14] OLIVEIRA, Andrielli Morais de. Avaliação do Desempenho de Concretos com Adições Minerais Quanto a Corrosão das Armaduras Induzida por Cloretos. 2007. 276 f. Dissertação (Mestrado em Engenharia Civil) Escola de Engenharia. Goiânia. 2007.

[15] PETRY, Simone Bassan. Estudo da Permeabilidade à Água na Camada de Cobrimento de Protótipos de Concretos com Altos Teores de Cinza Volante. 2004. 111 f. Dissertação (Mestrado em Engenharia Civil). Centro de Tecnologia. Santa Maria. 2004.

[16] AHMARUZZAMAN, M. A review on the utilization of fly ash. Progress in energy and Combustion Science. v. 36, n. 6, p. 327-363, 2010.

[17] SCHACKOW, Adilson. Efeito das Características Pozolânicas de Rejeitos de Tijolos nas Propriedades de Argamassas. Tese (Doutorado em Ciência e Engenharia de Materiais).Centro de Ciências Tecnológicas - CCT. 2015. Joinville.

[18] BRIZOLA, Rodrigo Matzenbacher. Microestrutura do Cobrimento de Concretos com Altos Teores de Escória e Cinza Volante Ativadas por Cimento Portland E Cal Hidratada. 2007. 179 f. Dissertação (Mestrado em Engenharia Civil) Centro de Tecnologia. Santa Maria. 2007.

[19] CAl, Xinhua et al. Abrasion erosion characteristics of concrete made with moderate heat Portland cement, fly ash and silica fume using sandblasting test. Construction and Building Materials. v. 127, n. 22, p. 804-814, 2016.

[20] KUMAR, Rakesh. Influence of Recycled Coarse Agregate Derived From Construction and Demolition Waste (CDW) on Abrasion Resistance of Pavement Concrete. Construction and Biulding Materials. v. 124, n. 11, p. 248255, 2017. 
[21] LAWRENCE, J. A comparative analysis of the wear characteristics of glazes generated on the ordinary Portland cement surface of concrete by means of $\mathrm{CO} 2$ and high power diode laser radiation. Wear. v. 257, n. 5-6, p. 590-598, mar. 2004.

[22] ACl Committee 302. ACl 302.1R-04-Guide for Concrete Floor and Slab Construction.

[23] BUEST Guilherme Teodoro; SILVA, Narciso G.; CAMPITELI, Vicente Coney. Dosagem de finos em concretos com agregados miúdos e graúdos britados. In: Congresso Brasileiro de Concreto, 47. 2005. São Paulo. Anais... São Paulo: Ibracon. 2005.

[24] STATSOFT, Inc. STATISTICA: data analysis software system. Version 7. 2007.

[25] Associação Brasileira de Normas Técnicas - ABNT. NBR 5738. Concreto: Procedimento para Moldagem e Cura de Corpos de Prova. Rio de Janeiro, 2016.

[26] CIENTEC - Fundação de Ciência e Tecnologia. Metodologia para Determinação de Resistência a Abrasão Superficial de Concreto. 2016.

[27] POSSAN, Edna; VENQUIARUTO, Simone Dorneles; DAL MOLIN, Denise Coutinho Carpena. Estudo da durabilidade de concretos com adição de cinza volante via metodologia de superfície de resposta. Revista Eletrônica Científica Inovação e Tecnologia. v. 1, n. 4, 2011.

[28] WITZKE, Franky Bruno. et al. Cost and property optimisation of concretes containing fly ash using mathematical and statistical strategies. International Symposium on Materials an International Conference, 8. 2017. Aveiro. Anais... Aveiro: SPM. 2017.

[29] ALMEIDA, I.R., GONCALVES, A.F.; VIEIRA, M. Concreto com cinzas volantes para pavimentação: Estudo da resistência a abrasão e da influência da cura úmida na durabilidade. In: $2^{\circ}$ Fórum Interamericano de Pavimentos de Concreto. Associação Brasileira de Cimento Portland, Rio de Janeiro: agosto de 1999. Anais..., p.239-251. 